\title{
La oxitocina en el tratamiento de los déficits sociales asociados a los trastornos del espectro autista
}

\author{
Carla Cachafeiro-Espino, Anna M. Vale-Martínez
}

Introducción. La implicación de la oxitocina en la conducta social de animales y humanos ha llevado a estudiar los efectos de su administración en el comportamiento y cognición social de pacientes con trastornos del espectro autista (TEA).

Objetivos. Revisar la investigación sobre el potencial terapéutico de la oxitocina en el tratamiento de los déficits sociales de la población con TEA y discutir las probables direcciones futuras de los estudios en este campo.

Desarrollo. Diversos trabajos han relacionado la oxitocina con la fisiopatología de los TEA. La mayoría de los estudios que han administrado oxitocina, generalmente por vía intranasal (24 $\mathrm{UI})$, ha observado mejoras significativas en el rendimiento social, sin detectar efectos secundarios destacables. No obstante, existen datos contradictorios debido a la heterogeneidad de las variables analizadas por los diferentes estudios, al uso de muestras heterogéneas y pequeñas o a la diferente duración de los tratamientos. Las limitaciones relacionadas con la falta de comprensión de los mecanismos de acción de la oxitocina y la diversidad sintomatológica de los TEA dificultan el establecimiento de este péptido como tratamiento de los pacientes autistas. Estudios recientes destacan la conveniencia de explorar el efecto de la combinación del tratamiento de oxitocina con programas conductuales de intervención en habilidades sociales, así como la potenciación de la secreción endógena de oxitocina.

Conclusiones. Los efectos de la administración de oxitocina resultan prometedores en relación con el tratamiento de los déficits sociales en individuos con TEA. Estudios futuros deberían facilitar la comprensión de las vías de acción de la oxitocina y el establecimiento de pautas óptimas de tratamiento.

Palabras clave. Autismo. Cognición social. Conducta social. Interacción social. Oxitocina. Revisión. Tratamiento.

\section{Introducción}

El uso de la hormona oxitocina para la potenciación de las habilidades sociales ha crecido exponencialmente en los últimos 10 años. Algunos estudios han sugerido que podría producir efectos terapéuticos en individuos que, por padecer algún trastorno psiquiátrico como la depresión, la esquizofrenia, el autismo o el estrés postraumático, presentan dificultades en las relaciones sociales [1]. La oxitocina es un neuropéptido que se sintetiza principalmente en las neuronas magnocelulares de los núcleos paraventricular y supraóptico del hipotálamo, para ser transportada por los axones de las neuronas hipotalámicas hasta la hipófisis posterior, donde se almacena y segrega al torrente sanguíneo [2]. No obstante, su potencial terapéutico se centra en la función neuromoduladora que ejerce en el sistema nervioso central, donde afecta a diferentes regiones cerebrales implicadas tanto en la percepción y cognición sociales como en la regulación emocional, que incluyen la amígdala, el hipocampo, el núcleo accumbens o el área tegmental ventral [3,4]. Además, algunos estudios indican que la oxitocina está rela- cionada con ciertas conductas sociales observadas en roedores, como el reconocimiento y la memoria social, el vínculo sexual y maternal, y la agresividad $[4,5]$. Asimismo, las investigaciones con voluntarios sanos parecen sugerir que este péptido podría ejercer un papel relevante en la conducta social humana. Por ejemplo, se ha observado que la administración exógena de oxitocina incrementa el tiempo de fijación en la mirada [6-8], facilita la inferencia de estados emocionales en los demás [9], aumenta la confianza ajena [10], potencia la codificación y, en consecuencia, el recuerdo de estímulos sociales positivos [11], y atenúa las evaluaciones negativas ante caras indicadoras de miedo [12].

En este contexto, recientemente se ha iniciado el estudio de la administración de oxitocina como tratamiento de trastornos que implican déficits en la interacción social, entre los que destacan los trastornos del espectro autista (TEA), caracterizados por presentar déficits en la comunicación y la interacción sociales, y patrones repetitivos y restringidos de conductas e intereses [13]. Concretamente, las disfunciones sociales de los TEA se definen a partir de déficits en distintos dominios, como la re-
Departamento de Psicobiología y Metodología de las Ciencias de la Salud. Instituto de Neurociencias. Universitat Autònoma de Barcelona. Bellaterra, Barcelona, España.

Correspondencia:

Dra. Anna María Vale Martínez. Departamento de Psicobiología y Metodología de las Ciencias de la Salud. Edificio B. Calle de la Fortuna. Universitat Autònoma de Barcelona. E-08193 Bellaterra (Barcelona).

Fax: +34 935812001

E-mail:

anna.vale@uab.cat

Declaración de intereses: Las autoras manifiestan la inexistencia de conflictos de interés en relación con este artículo.

Agradecimientos: Dr. Ferran Balada, por la revisión del manuscrito y sus valiosas sugerencias.

Aceptado tras revisión externa: 08.09.15.

Cómo citar este artículo: Cachafeiro-Espino C, ValeMartínez AM. La oxitocina en el tratamiento de los déficits sociales asociados a los trastornos del espectro autista. Rev Neurol 2015; 61: 421-8.

(c) 2015 Revista de Neurología 
ciprocidad socioemocional (disposición reducida a compartir intereses o dificultades para iniciar una conversación o responder a ella), la comunicación no verbal (anomalías en el contacto ocular o el lenguaje corporal), y el desarrollo y mantenimiento de relaciones sociales (dificultades para compartir juegos o hacer amigos) [13]. A pesar de la desadaptación que pueden generar estos síntomas en el individuo, aún no se ha aprobado un fármaco que resulte efectivo en la mejora de los déficits de interacción social característicos de dichos trastornos [14]. Así pues, es patente la necesidad de desarrollar algún tratamiento que facilite la adaptación de estos sujetos a su entorno social y, en consecuencia, mejore su calidad de vida. En este sentido, se ha sugerido que la oxitocina podría ser capaz de revertir algunos déficits sociales en personas con TEA, por ejemplo, incrementando el contacto ocular, facilitando la motivación social, mejorando las atribuciones que hacen de los demás o incrementando la empatía [15].

Este trabajo se propone revisar el estado actual de la investigación sobre el potencial terapéutico de la oxitocina en el tratamiento de los déficits sociales de la población con TEA, y discutir las direcciones futuras que deberían tomar los estudios en este campo. Con este objetivo en mente, se revisan los datos relativos a la relación entre la oxitocina y los TEA, los efectos de la administración de oxitocina en pacientes con TEA, los parámetros de tratamiento en términos de vías de administración y dosis óptimas, y las consideraciones a tener en cuenta en esta línea de investigación, así como alternativas de estudio no completamente desarrolladas hasta el momento.

\section{Relación entre la oxitocina y los TEA}

Aunque todavía no se ha determinado por completo la etiología de los TEA, está ampliamente aceptado que los factores genéticos desempeñan un papel importante en el desarrollo de dichos trastornos [16]. Diversos estudios han asociado ciertos polimorfismos del gen del receptor de la oxitocina a las poblaciones con TEA [17-21] o a alteraciones en la conducta social en humanos [22]. En cuanto a los niveles de oxitocina, Modahl et al [23] realizaron un estudio pionero que comparaba la oxitocina en el plasma sanguíneo de niños con desarrollo normal y con TEA, y observaron que estos últimos mostraban niveles periféricos de oxitocina significativamente menores. Estos resultados fueron replicados posteriormente por Andari et al [24] en una muestra adulta. También, Green et al [25] realizaron un estudio similar al de Modahl et al [23], y en- contraron que los niños con TEA no sólo presentaban niveles menores de oxitocina en plasma que los niños normales, sino que tenían diferentes niveles de una forma precursora de la oxitocina, la OXT-T. Por otro lado, en un estudio reciente [22] se observó que las diferencias en las concentraciones sanguíneas de oxitocina no estaban asociadas únicamente a los TEA, sino que correlacionaban con el rendimiento en pruebas de teoría de la mente y comunicación social, tanto en sujetos con TEA como en sus hermanos y otros niños no afectados, por lo que podrían contribuir a las diferencias individuales en el funcionamiento social humano.

No obstante, otro estudio reciente [26] -que a diferencia de los anteriores incluía una muestra de sujetos femeninos equiparable a la de sujetos masculinos- no encontró diferencias significativas entre las concentraciones de oxitocina en plasma de preadolescentes y adolescentes con TEA y el grupo control con desarrollo típico. En vez de ello, se hallaron diferencias significativas en los niveles periféricos de oxitocina en función del sexo; tanto las chicas con TEA como las del grupo control presentaban mayores concentraciones de oxitocina en plasma. Estos resultados sugieren que el sexo podría ser una variable relevante que se debería tener en cuenta en el estudio de la oxitocina como tratamiento. De hecho, Groppe et al [3] realizaron un estudio con una muestra exclusivamente femenina sobre la influencia de la oxitocina en la 'saliencia' de estímulos socialmente relevantes y detectaron efectos opuestos a los que habían sido notificados anteriormente con muestras masculinas. Estos datos, lejos de aportar pesimismo, dan soporte a la relación entre la oxitocina y los TEA, sobre todo si tenemos en cuenta la menor probabilidad de padecer TEA que presenta la población femenina $[27,28]$.

Otros datos que implican a la oxitocina en los TEA provienen de los estudios con animales. En primates, se ha descrito que los receptores de oxitocina se localizan en regiones colinérgicas que modulan la atención visual y auditiva, lo que sugiere un papel relevante en el procesamiento social sensorial [29]. También, Modi y Young [30] recogen diversas evidencias que muestran que ratones con alteraciones en el sistema de la oxitocina presentan anomalías de la conducta social en múltiples paradigmas conductuales. Por ejemplo, se ha observado que la supresión del gen que codifica el receptor de la oxitocina da lugar a un modelo conductual que mimetiza algunos síntomas característicos de los individuos con TEA, como deterioro de la flexibilidad cognitiva, socialización anormal y reducción de la ansiedad por separación de la madre [31]. Ade- 
más, Teng et al [32] hallaron que la administración aguda de oxitocina intraperitoneal producía efectos prosociales en dos modelos de TEA en ratones (BALB/cByJ, C58/J) que presentaban disfunciones en la interacción y la comunicación social y comportamientos estereotipados. Por otra parte, se ha descrito también en ratones que, durante el parto, se produce una inhibición gabérgica que está mediada por la oxitocina materna y que atenúa la patogenia del autismo en la prole [33].

\section{Efectos de la oxitocina administrada a poblaciones con TEA}

Los primeros estudios en este campo evaluaron la administración aguda de oxitocina. Así, Hollander et al [34] analizaron los efectos de una única inyección intravenosa de oxitocina, con una dosis que incrementaba gradualmente de 10 a $700 \mathrm{~mL} / \mathrm{h}$ a lo largo de cuatro horas, sobre la comprensión del discurso afectivo en 15 mujeres y hombres con TEA. Los resultados de este estudio indicaron que la oxitocina facilitaba el procesamiento emocional y la retención de información social en comparación con la administración de placebo. A pesar de que los resultados de este estudio fueron positivos, las investigaciones posteriores han utilizado la administración intranasal en vez de la intravenosa, por motivos que se recogen más adelante. En esta línea, Guastella et al [35] reclutaron a 16 jóvenes con TEA que fueron asignados al azar a recibir 18 o 24 UI de oxitocina o placebo. En este caso se observó que ambas dosis mejoraron la habilidad de los sujetos para asignar un estado emocional a diferentes expresiones faciales basándose en la región de los ojos, en comparación con el grupo placebo. Por otro lado, Andari et al [24] investigaron los efectos de la oxitocina intranasal (24 UI) sobre la conducta social de 13 pacientes con TEA en un juego de interacción con compañeros virtuales, en comparación con una condición placebo y el comportamiento de sujetos sanos. Los resultados revelaron que, después de la inhalación de oxitocina, los sujetos con TEA interactuaban predominantemente con el compañero de juego que había mostrado una actitud cooperativa, y mostraban mayores niveles de confianza y preferencia por éste. En el mismo estudio también se evaluaron los movimientos oculares de los pacientes con TEA ante la visualización de fotografías de caras, y observaron que la oxitocina aumentó el tiempo que éstos pasaban mirando la región de los ojos. Asimismo, un estudio muy reciente de Auyeung et al [8] ha demostrado, en una muestra de 37 hombres adultos con diagnóstico de autismo o síndrome de Asperger, que una aplicación intranasal de oxitocina (24 UI) aumenta el contacto ocular durante una interacción real con los investigadores, a diferencia de estudios precedentes que utilizaban interacciones virtuales o fotografías. En cambio, Domes et al [36] realizaron un estudio con 14 hombres con síndrome de Asperger y 14 hombres control en el que emparejaban estímulos en función de su parecido en una condición social (los estímulos eran caras) y una condición no social (los estímulos eran casas). Los participantes recibieron 24 UI de oxitocina o placebo intranasalmente antes de ejecutar la tarea, pero no se observaron diferencias significativas en el rendimiento de los sujetos en función de si habían recibido una $\mathrm{u}$ otra substancia. A pesar de ello, se observó un aumento de la actividad de la amígdala derecha ante los estímulos faciales en los individuos con TEA que habían recibido oxitocina en comparación con el placebo. Sobre la misma línea, Gordon et al [37] se propusieron valorar los cambios inducidos por la oxitocina intranasal en la actividad cerebral de 17 niños y adolescentes con TEA ante la evaluación de imágenes con significado social (ojos) y no social (coches). En este estudio se observó que la oxitocina aumentó la actividad en los circuitos neurales relacionados con la recompensa social, la motivación social y la teoría de la mente (por ejemplo, estriado, corteza orbitofrontal, surco temporal superior posterior) ante un test de lectura de la mente en los ojos. Un estudio reciente [38] ha ido más allá, ya que ha evaluado la habilidad para inferir emociones sociales de otros bajo condiciones que no incluyen señales sociales directas, y ha demostrado que la administración de oxitocina (24 UI intranasal) mejora dicha conducta en una muestra de 20 hombres con TEA con alto funcionamiento intelectual, así como su correlato neural, es decir, aumenta la actividad de la ínsula anterior, la cual se halla disminuida originalmente en individuos con TEA.

Aunque una única dosis de oxitocina parece capaz de potenciar la respuesta social de los individuos con TEA, resulta más interesante la evaluación de su administración continuada, ya que permite el análisis de sus efectos a largo plazo y sería lo habitual al utilizarla como tratamiento. En un primer informe de caso único [39] se realizó un seguimiento de los efectos de la administración intranasal de 8 UI de oxitocina durante un período de dos meses a una joven autista de 16 años. Los resultados revelaron mejoras en la comunicación social y la irritabilidad de la adolescente, a partir del juicio clínico e informes paternos. Otro estudio [40] seleccionó 19 hombres y adolescentes con síndrome de Asperger para admi- 
nistrarles 24 UI de oxitocina, dos veces al día, durante seis semanas. Aunque no se encontraron cambios significativos en medidas generales de cognición social o comportamientos repetitivos, se detectaron mejoras en la prueba de reconocimiento de emociones y de calidad de vida. Más tarde, Tachibana et al [41] realizaron un estudio con ocho adolescentes con TEA tratados con oxitocina intranasal durante seis meses, incrementando la dosis cada dos meses $(8,16$ y $24 \mathrm{UI}$ ) e incluyendo un período de placebo de una o dos semanas antes de cada cambio de dosis. Los resultados, a partir de pruebas psicométricas, revelaron mejoras significativas en la comunicación y la interacción social en seis de los ocho participantes. Anagnostou et al [42] evaluaron los efectos de diferentes dosis de oxitocina intranasal en 15 niños y adolescentes con TEA en cuatro dominios distintos: cognición social, conducta social, comportamientos repetitivos y ansiedad. Después del tratamiento se observaron mejoras en todos los dominios evaluados, que se mantuvieron durante tres meses. Finalmente, Dadds et al [43] realizaron una intervención a corto plazo, a lo largo de cuatro días, con 38 niños y adolescentes con TEA, en la que les administraban intranasalmente 12 o 24 UI de oxitocina o placebo durante sesiones de entrenamiento en interacción entre padres e hijos. Se evaluaron las habilidades de interacción social, los comportamientos repetitivos, el reconocimiento de emociones y la adaptación general del comportamiento de los jóvenes y, contrariamente a lo esperado, no se encontraron mejoras significativas debidas a la aplicación de oxitocina. Esta discrepancia podría deberse a que el tratamiento era mucho más breve que en los estudios precedentes o bien a diferencias metodológicas, como la pauta de administración o la diversidad en las medidas utilizadas para valorar los resultados.

Aunque, en general, la oxitocina proporciona resultados positivos en el comportamiento social, las discrepancias citadas dificultan la comparación de los resultados y el establecimiento de conclusiones firmes acerca de sus beneficios terapéuticos.

\section{Parámetros del tratamiento}

\section{Vía de administración}

Como se ha comentado, existe una notable tendencia al uso de la vía intranasal para administrar oxitocina. De hecho, el uso de esta vía de administración se ha incrementado de manera significativa en los últimos años, dado que se ha observado que podría evitar la barrera hematoencefálica y alargar la vida media de la oxitocina en el organismo. A pesar de que no se conoce con certeza el mecanismo de acción de la oxitocina administrada por vía intranasal, datos recientes indican que podría acceder directamente desde el epitelio nasal a diferentes regiones del sistema nervioso, tanto olfatorias como otras, a partir de los espacios perineurales y perivasculares $[44,45]$. Por otra parte, aunque los estudios que han administrado oxitocina por vía intravenosa también han encontrado mejoras significativas en la conducta social y los comportamientos repetitivos de los niños con TEA $[34,46]$, obviamente la vía intranasal resulta una alternativa más cómoda y menos invasiva que la intravenosa, y más teniendo en cuenta que el tratamiento de los TEA podría estar dirigido a poblaciones pediátricas [47].

\section{Dosis óptima}

En cuanto al establecimiento de la dosis óptima, son pocos los ensayos clínicos que han utilizado más de una dosis, de manera que los datos sobre cuál es la más efectiva son limitados [48]. De los estudios revisados en el presente trabajo, sólo uno ha comparado diferentes dosis para averiguar la máxima tolerada por niños con TEA [42]. En este estudio se evaluaron cuatro dosis diferentes en función del peso de los sujetos $(0,2-0,4 \mathrm{UI} / \mathrm{kg})$, y la más alta es la que se ha utilizado con más frecuencia (correspondería a $24 \mathrm{UI} / 60 \mathrm{~kg}$ ). Por otra parte, parece ser que el tratamiento continuado con oxitocina se tolera de manera aceptable, pues los efectos secundarios, si están presentes, son calificados como moderados $[40,49]$. Sin embargo, algunos sujetos han comunicado agitación, irritabilidad, aumento de energía, alergia, fatiga leve, dolor de cabeza o temblores en las piernas. Así, aunque se ha demostrado que la administración de 24 UI es segura y efectiva, se desconoce si dosis más altas podrían conferir mayores beneficios con parámetros de seguridad aceptables [42]. Por lo tanto, con estas observaciones se evidencia la necesidad de realizar estudios que comparen un rango de dosis, los cuales apuntarán datos sobre la relación dosis-respuesta en la conducta social de los sujetos con TEA y los efectos secundarios, aspecto importante debido a que la oxitocina presenta afinidad cruzada con los receptores de la vasopresina [48].

\section{Consideraciones en el estudio del potencial terapéutico de la oxitocina en los TEA}

Una importante limitación que surge en relación con la oxitocina como posible tratamiento, derivada de 
la novedad de este campo de investigación, es que no existe una comprensión completa de cómo ejerce sus efectos en el cerebro ni de qué variables pueden alterar su funcionamiento ni a través de qué mecanismos. En este contexto, resultaría interesante relacionar los efectos de la oxitocina con su actividad en el sistema nervioso central, a partir de las principales regiones cerebrales sobre las que actúa y su interacción con otros sistemas de neurotransmisión. De esta manera, podrían establecerse biomarcadores que permitirían anticipar la respuesta a la administración de oxitocina. Parker et al [22] proponen el uso de las concentraciones de oxitocina en plasma y el estudio genético de los polimorfismos del gen del receptor de la oxitocina como biomarcadores capaces de predecir sus efectos en diferentes medidas relacionadas con las habilidades sociales. En este sentido, los estudios realizados hasta el momento sobre el sistema oxitoninérgico han mostrado que existen variaciones individuales en los niveles basales de oxitocina y en la reactividad del sistema en función de diferentes variables.

Algunos autores han sugerido que el sistema de la oxitocina podría estar condicionado por el sexo del sujeto, ya que, como se ha comentado, se han encontrado diferencias en las concentraciones plasmáticas de oxitocina de hombres y mujeres [26], y efectos opuestos de la administración de la oxitocina según si la muestra era femenina o masculina [3]. Asimismo, parece que la influencia de la oxitocina depende de variables relacionadas con la conducta social presentada por los individuos antes del tratamiento. Por ello, en el tratamiento de los TEA resulta relevante tener en cuenta la diversidad de manifestaciones clínicas de estos pacientes, en cuanto al tipo de síntomas presentados y la gravedad de los déficits sociales o su funcionamiento intelectual [50]. Algunos estudios han detectado diferencias en los efectos de la oxitocina según la dificultad percibida en una tarea de reconocimiento de emociones, tanto en una muestra de hombres sanos [9] como de jóvenes con TEA [35], y según las puntuaciones obtenidas en un autoinforme de amabilidad [3]. Los estudios con animales también corroboran esta relación, pues se observan diferencias en los efectos del tratamiento en función de la predisposición a interactuar con el cuidador en una muestra de macacos recién nacidos [51]. Es necesario que futuros estudios persigan una comprensión sólida de cómo las diferencias individuales pueden alterar los efectos de la administración de oxitocina, para poder evaluar tanto los efectos del tratamiento como sus riesgos y beneficios potenciales. Para ello, hay que atender tanto a las influencias de los factores gené- ticos como a los condicionantes del entorno, ya que las experiencias sociales y los acontecimientos vitales estresantes pueden afectar a largo plazo el sistema de la oxitocina [47].

En el intento de comprender mejor el funcionamiento del sistema de la oxitocina, Churchland y Winkielman [52] sugieren el estudio de los efectos de esta hormona en términos de procesos básicos, como la ansiedad o la motivación social, en lugar de atribuirle la capacidad de modular conductas sociales complejas, como la empatía o la confianza, que probablemente dependen de numerosos factores. En este contexto puede resultar relevante el modelo explicativo elaborado por Bartz et al [53], que propone que los efectos de la oxitocina sobre la cognición y la conducta social de los sujetos están regulados por mecanismos como la reducción de la ansiedad, el aumento de la motivación de afiliación o la 'saliencia' de los estímulos sociales. A su vez, este modelo integra diferentes moduladores que pueden condicionar los mecanismos de acción de la oxitocina [52], relacionados con diferencias individuales (competencia social o rasgos de personalidad) y con variables ambientales (dificultad de la tarea o valencia de los estímulos).

Por otra parte, dado el inicio temprano de los trastornos autistas [13] y la posible implicación de la oxitocina en su fisiopatología, sería interesante que los futuros estudios estableciesen qué relaciones existen entre la etiología de los TEA y las alteraciones en el sistema oxitoninérgico, y si la administración temprana de oxitocina puede alterar significativamente el desarrollo del trastorno. En este sentido, un estudio reciente ha analizado los efectos de la oxitocina en macacos recién nacidos y ha obtenido resultados positivos en su conducta social [51]. Asimismo, Tyzio et al [33] sugieren un efecto neuroprotector de la oxitocina liberada durante el parto en ratones, que va más allá de los efectos clásicamente descritos de establecimiento de vínculos. No obstante, debería considerarse un posible efecto perjudicial de la oxitocina sobre la maduración cerebral porque se ha observado que la administración crónica intranasal podría reducir la expresión de receptores de oxitocina y el comportamiento social en ratones normales [54]. Así pues, una administración intermitente podría ser preferible a una administración crónica.

Finalmente, el estudio de la oxitocina en la potenciación de las habilidades sociales abre paso a interesantes líneas de investigación, como la combinación del tratamiento de oxitocina con programas de intervención psicológica. Debido al hecho de que el contexto es crítico para el éxito de las te- 
rapias con oxitocina [53], algunos autores han argumentado que la administración del péptido junto con terapias psicológicas es crucial para obtener mejoras generalizadas y a largo plazo en las conductas sociales afectadas en los jóvenes con TEA [55]. Así, el uso de la oxitocina podría facilitar la iniciativa, el reconocimiento y la cognición social en el momento del abordaje educativo-conductual [56]. Concretamente, Modi et al [30] sugieren que los efectos de la oxitocina sobre la 'saliencia' de los estímulos sociales, el incremento del contacto ocular y la comprensión de las expresiones faciales podrían facilitar el aprendizaje en los programas de entrenamiento de habilidades sociales y, en consecuencia, mejorar la calidad de vida de los pacientes y sus familiares [49]. Por otra parte, otra línea de investigación que surge en el estudio de la oxitocina, al tratarse de una sustancia que se libera de forma natural en nuestro organismo, es la potenciación de su secreción endógena. Esta aproximación solventaría una de las limitaciones importantes de las terapias actuales con oxitocina, como son la eficaz penetración en el cerebro y la activación de los receptores. Además, resulta aún más relevante si se tiene en cuenta el inicio temprano de los TEA, ya que podría suponer una forma de intervención precoz no demasiado invasiva que permitiría alterar el curso del trastorno y prevenir la expresión de algunos comportamientos típicos de estos individuos en la edad adulta [57]. Así, aunque esta opción no ha sido muy explorada hasta el momento, algunos estudios han notificado que la acupuntura de estimulación eléctrica transcutánea (una variante de la acupuntura tradicional) [58] y la escucha de música relajante durante el período postoperatorio de una intervención coronaria [59] incrementaron las concentraciones de oxitocina en sangre de forma significativa en comparación con los sujetos que no habían recibido dichas intervenciones.

\section{Conclusiones}

Los datos que sugieren la posible existencia de una alteración en el sistema oxitoninérgico en la población con TEA proporcionan una base para considerar la oxitocina en el tratamiento de estos pacientes. Además, los estudios realizados hasta el momento ponen de manifiesto que la administración de oxitocina mejora algunos de los déficits característicos de los TEA, como el reconocimiento de las emociones y el contacto ocular [49]. Los estudios multidosis sugieren que el tratamiento con oxitocina intranasal, en dosis no superiores a 24 UI, es eficaz y se- guro a la hora de promover cambios en la conducta social de los sujetos que presentan TEA. Estos datos comportan cierto optimismo con respecto al potencial terapéutico de la oxitocina y justifican la necesidad de continuar con esta línea de investigación con el fin de consolidar una nueva forma de tratamiento que permita mejorar la calidad de vida de la población autista.

No obstante, existe una cierta variabilidad en los resultados de los estudios en humanos que podrían ser atribuidas a diferencias en dosis, duración de los estudios, edad y muestras pequeñas y heterogéneas [60]. Así pues, para optimizar las pautas de tratamiento, será necesario que se resuelvan algunas de estas limitaciones. Por una parte, se requieren estudios que comparen diferentes dosis de oxitocina, durante períodos de tiempo más largos y que valoren la permanencia de los resultados positivos a largo plazo e incluso más allá del tiempo de tratamiento. Además, se necesitan muestras más amplias, que cuenten con niñas y mujeres [49] y con individuos con discapacidad intelectual, una condición habitualmente comórbida en los TEA. En este sentido, los individuos con un funcionamiento intelectual inferior han sido tradicionalmente excluidos de los ensayos con oxitocina debido a la dificultad para administrarles tareas sociocognitivas, cuyas medidas requieren respuestas verbales [61]. También sería necesario establecer consenso respecto a las medidas de resultados que permitan evaluar de forma objetiva y fiable los efectos del tratamiento con oxitocina. Por otro lado, se espera que los estudios futuros contribuyan a comprender mejor los mecanismos de acción de la oxitocina, para lo que se requieren estudios en animales y humanos en paralelo, así como las variables que pueden modular las acciones de la oxitocina, tanto a nivel individual como del entorno. En este contexto, sería interesante establecer biomarcadores que permitan prever el efecto del tratamiento y ajustarlo a las necesidades de cada persona. Finalmente, resultan atractivas las líneas de investigación que aluden al uso de la oxitocina como coadyuvante de intervenciones psicológicas sobre las habilidades sociales o la potenciación de la secreción endógena de oxitocina.

\section{Bibliografía}

1. Bakermans-Kranenburg MJ, Van Ijzendoorn MH. Sniffing around oxytocin: review and meta-analyses of trials in healthy and clinical groups with implications for pharmacotherapy. Transl Psychiatry 2013; 3: e258.

2. Burbach JPH, Young LJ, Russell JA. Oxytocin: synthesis, secretion, and reproductive functions. In Neill JD, ed. Knobil and Neill's physiology of reproduction. New York: Elsevier; 2006. p. 3055-128. 
3. Groppe SE, Gossen A, Rademacher L, Hahn A, Westphal L, Gründer G, et al. Oxytocin influences processing of socially relevant cues in the ventral tegmental area of the human brain Biol Psychiatry 2013; 74: 172-9.

4. Febo M, Ferris CF. Oxytocin and vasopressin modulation of the neural correlates of motivation and emotion: results from functional MRI studies in awake rats. Brain Res 2014; 1580: 8-21.

5. Lee HJ, Macbeth AH, Pagani JH, Young WS. Oxytocin: the great facilitator of life. Prog Neurobiol 2009; 88: 127-51.

6. Guastella AJ, Mitchell PB, Dadds MR. Oxytocin increases gaze to the eye region of human faces. Biol Psychiatry 2008; 63: 3-5.

7. Domes G, Steiner A, Porges SW, Heinrichs M. Oxytocin differentially modulates eye gaze to naturalistic social signals of happiness and anger. Psychoneuroendocrinology 2013; 38 1198-202.

8. Auyeung B, Lombardo MV, Heinrichs M, Chakrabarti B, Sule A, Deakin JB, et al. Oxytocin increases eye contact during a real-time, naturalistic social interaction in males with and without autism. Transl Psychiatry 2015; 5: e507.

9. Domes G, Heinrichs M, Miguel A, Berger C, Herpetzs SC. Oxytocin improves 'mind-reading' in humans. Biol Psychiatry 2007; 61: 731-3.

10. Kosfeld M, Heinrichs M, Zak PJ, Fischbacher U, Fehr E. Oxytocin increases trust in humans. Nature 2005; 435: 673-6.

11. Guastella AJ, Mitchell PB, Mathews F. Oxytocin enhances the encoding of positive social memories in humans. Biol Psychiatry 2008; 64: 256-8.

12. Petrovic P, Kalisch R, Singer T, Dolan RJ. Oxytocin attenuates affective evaluations of conditioned faces and amygdala activity. J Neurosci 2008; 28: 6607-15.

13. Colegio Oficial de Psicólogos. Manual diagnóstico y estadístico de los trastornos mentales, 5 ed. Madrid: Editorial Médica Panamericana; 2014.

14. Posey D, Erickson C, McDougle C. Developing drugs for core social and communication impairment in autism. Child Adolesc Psychiatr Clin N Am 2008; 17: 787-ix.

15. Liu J, McErlean R, Dadds M. Are we yet? The clinical potential of intranasal oxytocin in psychiatry. Curr Psychiatry Rev 2012 8: $37-48$

16. Lintas C, Persico A. Autistic phenotypes and genetic testing state-of-the-art for the clinical geneticist. J Med Genet 2009; 46: 1-8.

17. Yamasue H. Function and structure in social brain regions can link oxytocin-receptor genes with autistic social behavior Brain Dev 2013; 35: 111-8.

18. Wu S, Jia M, Ruan Y, Liu J, Guo Y, Shuang M, et al. Positive association of the oxytocin receptor gene (OXTR) with autism in the Chinese Han population. Biol Psychiatry 2005; 58: 74-7.

19. Jacob S, Brune C, Carter CS, Leventhal BL, Lord C, Cook Jr EH. Association of the oxytocin receptor gene (OXTR) in Caucasian and adolescents with autism. Neurosci Lett 2007; 417: 6-9.

20. Lerer E, Levi S, Salomon S, Darvasi A, Yirmiya N, Ebstein RP. Association between the oxytocin receptor (OXTR) gene and autism: relationship to Vineland Adaptative Behavior Scales and cognition. Mol Psychiatry 2008; 13: 980-8.

21. LoParo D, Waldman ID. The oxytocin receptor gene (OXTR) is associated with autism spectrum disorder: a meta-analysis. Mol Psychiatry 2015; 20: 640-6.

22. Parker KJ, Garner JP, Libove RA, Hyde SA, Hornbeak KB, Carson DS, et al. Plasma oxytocin concentrations and OXTR polymorphisms predict social impairments in children with and without autism spectrum disorder. Proc Natl Acad Sci U S A 2014; 111: 12258-63.

23. Modahl C, Green L, Fein D, Morris M, Waterhouse L, Feinstein C, et al. Plasma oxytocin levels in autistic children. Biol Psychiatry 1998; 43: 270-7.

24. Andari E, Duhamel JR, Zalla T, Herbrecht E, Leboyer M, Sirigu A. Promoting social behavior with oxytocin in highfunctioning autism spectrum disorders. Proc Natl Acad Sci U S A 2010; 107: 4389-94.

25. Green L, Fein D, Modahl C, Feinstein C, Waterhouse L,
Morris M. Oxytocin and autistic disorder: alterations in peptide forms. Biol Psychiatry 2001; 50: 609-13.

26. Miller M, Bales KL, Taylor SL, Yoon J, Hostetler CM, Carter CS et al. Oxytocin and vasopressin in children and adolescents with autism spectrum disorders: sex differences and associations with symptoms. Autism Res 2013; 6: 91-102.

27. Fombonne E. Epidemiology of autistic disorder and other pervasive developmental disorders. J Clin Psychiatry 2005; 66 (Suppl 10): S3-8.

28. Whiteley P, Todd L, Carr K, Shattock P. Gender ratios in autism, Asperger syndrome and autism spectrum disorder. Autism Insight 2010; 2: 17-24.

29. Freeman SM, Inoue K, Smith AL, Goodman MM, Young LJ. The neuroanatomical distribution of oxytocin receptor binding and mRNA in the male rhesus macaque (Macaca mulatta). Psychoneuroendocrinology 2014; 45: 128-41.

30. Modi ME, Young LJ. The oxytocin system in drug discovery for autism: animal models and novel therapeutic strategies. Horm Behav 2012; 61: 340-50.

31. Sala M, Braida D, Lentini D, Busnelli M, Bulgheroni E, Capurro V, et al. Pharmacologic rescue of impaired cognitive flexibility, social deficits, increased aggression, and seizure susceptibility in oxytocin receptor null mice: a neurobehavioral model of autism. Biol Psychiatry 2011; 69: 875-82.

32. Teng BL, Nonneman RJ, Agster KL, Nikolova VD, Davis TT, Riddick NV, et al. Prosocial effects of oxytocin in two mouse models of autism spectrum disorders. Neuropharmacology 2013; 72: 187-96.

33. Tyzio R, Nardou R, Ferrari DC, Tsintsadze T, Shahrokhi A Eftekhari S, et al. Oxytocin-mediated GABA inhibition during delivery attenuates autism pathogenesis in rodent offspring. Science 2014; 343(6171): 675-9.

34. Hollander E, Bartz J, Chaplin W, Phillips A, Sumner J, Soorya L, et al. Oxytocin increases retention of social cognition in autism. Biol Psychiatry 2007; 61: 498-503.

35. Guastella AJ, Einfeld SL, Gray KM, Rinehart NJ, Tonge BJ, Lambert TJ, et al. Intranasal oxytocin improves emotion recognition for youth with autism spectrum disorders. Biol Psychiatry 2010; 67: 692-4.

36. Domes G, Heinrichs M, Kumbier E, Grossman A, Hauenstein K, Herpetz SC. Effects of intranasal oxytocin on the neural basis of face processing in autism spectrum disorder. Biol Psychiatry 2013; 74: 164-71.

37. Gordon I, Van der Wyk BC, Bennett RH, Cordeaux C, Lucas MV, Eilbott JA, et al. Oxytocin enhances brain function in children with autism. Proc Natl Acad Sci U S A 2013; 110: 20953-8.

38. Aoki Y, Yahata N, Watanabe T, Takano Y, Kawakubo Y, Kuwabara H, et al. Oxytocin improves behavioural and neural deficits in inferring others' social emotions in autism. Brain 2014; 137: 3073-86

39. Kosaka H, Munesue T, Ishitobi M, Asano M, Omori M, Sato M, et al. Long-term oxytocin administration improves social behaviors in a girl with autistic disorder. BMC Psychiatry 2012; 12: 110.

40. Anagnostou E, Soorya L, Chaplin W, Bartz J, Halpern D, Wasserman $S$, et al. Intranasal oxytocin versus placebo in the treatment of adults with autism spectrum disorders: a randomized controlled trial. Mol Autism 2012; 3: 16

41. Tachibana M, Kagitani-Shimono K, Mohri I, Yamamoto T, Sanefuji W, Nakamura A, et al. Long-term administration of intranasal oxytocin is a safe and promising therapy for early adolescent boys with autism spectrum disorders. J Child Adolesc Psychopharmacol 2013; 23: 123-7.

42. Anagnostou E, Soorya L, Brian J, Dupuis A, Mankad D, Smile S, et al. Intranasal oxytocin in the treatment of autism spectrum disorders: A review of literature and early safety and efficacy data in youth. Brain Res 2014; 1580: 188-98.

43. Dadds MR, MacDonald E, Cauchi A, Williams K, Levy F, Brennan J. Nasal oxytocin for social deficits in childhood autism: a randomized controlled trial. J Autism Dev Disord 2014; 44: 521-31.

44. Illum L. Transport of drugs from the nasal cavity to the central nervous system. Eur J Pharm Sci 2000; 11: 1-18. 
45. Veening JG, Olivier B. Intranasal administration of oxytocin: behavioral and clinical effects, a review. Neurosci Biobehav Rev 2013; 37: 1445-65.

46. Hollander E, Novotny S, Hanratty M, Yaffe R, DeCaria CM, Aronowitz BR, et al. Oxytocin infusion reduces repetitive behaviors in adults with autistic and Asperger's disorders. Neuropsycopharmacology 2003; 28: 193-8.

47. Taylor AE, Lee H, Buisman-Pijlman FTA. Oxytocin treatment in pediatric populations. Front Behav Neurosci 2014; 8: 360.

48. MacDonald K, Feifel D. Helping oxytocin deliver: considerations in the development of oxytocin-based therapeutics for brain disorders. Front Neurosci 2013; 7: 35.

49. Preti A, Melis M, Siddi S, Vellante M, Doneddu G, Fadda R. Oxytocin and autism: a systematic review of randomized controlled trials. J Child Adolesc Psychopharmacol 2014; 2: 54-68.

50. Green JJ, Hollander E. Autism and oxytocin: new developments in translational approaches to therapeutics. Neurotherapeutics 2010; 7: 250-7.

51. Simpson EA, Sclafani V, Paukner A, Hamel AF, Novak MA, Meyer JS, et al. Inhaled oxytocin increases positive social behaviors in newborn macaques. Proc Natl Acad Sci U S A 2014; 111: 6922-7.

52. Churchland PS, Winkielman P. Modulating social behavior with oxytocin: how does it work? What does it mean? Horm Behav 2012; 61: 392-9.

53. Bartz JA, Zaki J, Bolger N, Ochsner KN. Social effects of oxytocin in humans: context and person matter. Trend Cogn Sci 2011; 15: 301-9.

54. Huang H, Michetti C, Busnelli M, Managò F, Sannino S, Scheggia D, et al. Chronic and acute intranasal oxytocin produce divergent social effects in mice. Neuropsychopharmacology 2014; 39: 1102-14.

55. Stravopoulos KMK, Carver LJ. Research review: social motivation and oxytocin in autism-implications for joint attention development and intervention. J Child Psychol Psychiatry 2013; 54: 603-18.

56. Ruggieri VL, Arberas CL. Abordajes terapéuticos en los trastornos del espectro autista. Rev Neurol 2015; 60 (Supl 1): S45-9.

57. Carter CS. Sex differences in oxytocin and vasopressin: implications for autism spectrum disorders? Behav Brain Res 2007; 176: 170-86

58. Zhang R, Jia MX, Zhang JS, Xu XJ, Shou XJ, Zhang XT, et al. Transcutaneous electrical acupoint stimulation in children with autism and its impact on plasma levels of argininevasopressin and oxytocin: a prospective single-blinded controlled study. Res Dev Disabil 2012; 33: 1136-46.

59. Nilsson U. Soothing music can increase oxytocin levels during bed rest after open-heart surgery: a randomised control trial. J Clin Nurs 2009; 18: 2153-61.

60. Young LJ, Barrett CE. Neuroscience. Can oxytocin treat autism? Science 2015; 347: 825-6.

61. Politte LC, Henry CA, McDougle CJ. Psychopharmacological interventions in autism spectrum disorder. Harv Rev Psychiatry 2014; 2: 76-92.

\section{Oxytocin in the treatment of the social deficits associated to autism spectrum disorders}

Introduction. The recent involvement of oxytocin in social behavior of animals and humans has motivated the study of its effects on the social behavior of individuals with autism spectrum disorders (ASD).

Aims. To review the current state of oxytocin studies concerning its therapeutic potential in treating social deficits of the ASD population, and to establish likely future directions to be taken by the studies in this field.

Development. Some studies have linked oxytocin to the pathophysiology of autistic disorders. Most studies that have administered oxytocin (mainly with intranasal administration of 24 IU) to ASD subjects have shown significant improvements in their social performance with acceptable safety parameters. However, there is controversial data as the outcome measures are widely dispersed, the samples are reduced and heterogeneous, and the treatment durations are different. The limitations related to the lack of understanding of the oxytocin's action mechanisms and the symptomatic heterogeneity of ASD are hampering progress towards the establishment of oxytocin as a treatment of ASD patients. Recent studies suggest the investigation of the combination of the oxytocin treatment with social skills training, and the enhancement of endogenous secretion of oxytocin.

Conclusion. The effects of oxytocin are promising regarding the treatment of social deficits in ASD individuals. Future studies should aim to facilitate understanding of the oxytocin's ways of action and to establish the optimal treatment regime.

Key words. Autism. Oxytocin. Review. Social behavior. Social cognition. Social interaction. Treatment. 